\title{
Structure Watch
}

\section{SIGNAL PEPTIDE RECOGNITION}

Most secretory and membrane proteins contain a signal peptide, which is recognized by the signal recognition particle (SRP) when newly synthesized polypeptide chains emerge from the ribosome. The signal peptide targets the SRP-ribosome nascent chain (RNC) complex to protein-translocating channels in the endoplasmic reticulum membrane in eukaryotes and in the plasma membrane in bacteria. Bacterial SRPs consist of 4.5S RNA and Ffh, which comprises an amino-terminal (N) domain, a GTPase (G) domain and a carboxy-terminal Met-rich (M) domain. The signal peptide was proposed to bind to the M domain, but a SRP structure with bound signal peptide has been lacking. Janda et al. produced a fusion protein of bacterial Ffh and a signal peptide connected by a flexible linker. The fusion protein oligomerizes in solution through the interaction between Ffh and signal peptides of different polypeptide chains. The X-ray structure of a dimeric complex was then determined. The signal peptide binds to a groove formed between $\alpha$-helices of the $M$ domain, and residues in the hydrophobic region of the signal peptide interact with hydrophobic residues in the groove. Furthermore, binding of the SRP to the RNC complex causes a large movement of the $\mathrm{N}$ and $\mathrm{G}$ domains with respect to the $\mathrm{M}$ domain. However, binding of signal peptide to the $\mathrm{M}$ domain alone is insufficient to induce this large rearrangement, and the linker between the $\mathrm{N}$ and $\mathrm{G}$ domains and the $\mathrm{M}$ domain is thought to couple signal peptide binding and ribosome binding.

ORIGINAL RESEARCH PAPER Janda, C. Y. et al. Recognition of a signal peptide by the signal recognition particle. Nature 4 Apr 2010 (doi:10.1038/nature08870)

\section{CELL-CELL INTERACTIONS}

p120 catenin mediates cell-cell adhesion by interacting with the conserved juxtamembrane domain (JMD) of cadherins. Uncoupling of the 120 catenin-JMD interaction or a reduction in 120 catenin levels increases cadherin internalization through an endocytic diLeu (LL) motif; the association of p120 catenin with JMD is proposed to sterically hinder the endocytic machinery from associating with cadherin. The authors determined the crystal structure of $\mathrm{p} 120$ catenin isoform $4 \mathrm{~A}$ in complex with the JMD core region of epithelial cadherin (E-cadherin). JMD electrostatically and hydrophobically interacts with $\mathrm{p} 120$ catenin armadillo repeats $1-5$. Structure-based mutagenesis studies in epithelial and neuronal cells showed that single-residue mutations in the JMD-binding site of $\mathrm{p} 120$ catenin are sufficient to uncouple p120 catenin from E-cadherin and neuronal cadherin, respectively. NMR studies identified dynamic and static binding interfaces between $\mathrm{p} 120$ catenin and JMD; the static binding site is mainly responsible for the specific interaction between p120 catenin and E-cadherin, whereas the dynamic binding site protects the LL motif from the endocytic machinery. 